\title{
Measurement of the Integral Intensity of Near Horizontal Muons with HAWC
}

\author{
Ahron Barber* \\ University of Utah \\ E-mail: ahron.barbereutah.edu \\ David Kieda \\ University of Utah \\ E-mail: dave.kieda@utah.edu
}

\section{R. Wayne Springer}

University of Utah

E-mail: wayne.springereutah.edu

\section{for the HAWC Collaboration}

For a complete author list see https://www.hawc-observatory.org/collaboration/icrc2019.php

The HAWC (High Altitude Water Cherenkov) gamma ray observatory has observed muons at nearly horizontal directions. The proximity of Sierra Negra and Pico de Orizaba volcanoes provides a range of depths with rock overburdens of up to approximately $30 \mathrm{~km}$ water equivalent. In the arrival directions not obstructed by the nearby volcanoes of Sierra Negra and Pico de Orizaba the depth traversed by muons is that of the Earth's atmosphere for horizontal trajectories of approximately $0.2 \mathrm{~km}$ water equivalent. We have implemented a muon candidate selection procedure using line and plane finding algorithms to identify isolated muon tracks (lines) propagating near horizontally with the speed of light among a large background of extensive air shower (planes). This selection procedure also reconstructs the muon's arrival direction. We have modified existing HAWC simulation software and developed geometry-based calculations to estimate detector acceptance and reconstruction resolution of arrival direction to determine the exposure as a function of rock overburden. Using this exposure and the rate of observed muons as a function of arrival direction we have performed a measurement of muon flux as a function of depth from $0.2 \mathrm{~km}$ water equivalent up to $30 \mathrm{~km}$ water equivalent. Muons that penetrate depths of greater than $3 \mathrm{~km}$ water equivalent are likely to have had initial energies in excess of $1 \mathrm{TeV}$. Our measurements are consistent with previous experiments that have observed high zenith angle (near horizontal) muon flux at or near sea level as well as muon flux measurements as a function of depth in deep underground facilities.

36th International Cosmic Ray Conference -ICRC2019-

July 24th - August 1st, 2019

Madison, WI, U.S.A.

\footnotetext{
* Speaker.
} 


\section{Introduction}

Studying muons underground began only a few years after they were discovered. The muon's ability to penetrate significant amounts of matter enabled experiments in deep mines or underwater to measure the flux of cosmic ray induced muons as a function of depth. Those experiments obtained varying depths for their measurements by looking through the rock overburden at various zenith angles. The initial muon energy is related to the range or overburden depth traversed. Measuring the flux of muons through a given depth is related to the integral intensity above a minimum energy [1]. For this work, we compare our results to E.V. Bugaev et al., who compiled the previous experimental results into one integral intensity measurement to investigate the prompt muon component from cosmic rays [2]. In this proceeding, we present an integral intensity measurement of muons as a function of rock overburden up to $10 \mathrm{~km}$.m.w.e. using HAWC and surrounding volcanoes.

HAWC the High Altitude Water Cherenkov detector is primarily a Gamma and Cosmic Ray detector located at $4100 \mathrm{~m}$ a.s.l. on the volcano Sierra Negra in Mexico. This paper is reporting on the effort to study one of two ancillary science topics of interest to HAWC; both of which look for muons traveling horizontally through the detector. H. León Vargas is presenting in this conference a separate analysis to identify near-horizontal muons in HAWC specifically looking for neutrino events from Pico de Orizaba [3]. We are focused on understanding the near-horizontal muons to investigate the Cosmic Ray composition near the knee. Using muons to study the composition is done by looking at muon bundles (i.e. multiple simultaneous muons) which have been done most recently by the Decor-Nevod detector [4]. HAWC has a significantly larger water volume, and we are making progress in providing a complementary result. The initial step, presented here, is to look at the single muon integral intensity and HAWC's effective area. We also identify possible roadblocks for multiple muon identification.

\section{Muon Identification}

HAWC is composed of 300 Water Cherenkov Detectors (WCD) densely packed over 22,000 $m^{2}$ to sample the Extensive Air Shower front for Gamma and Cosmic Ray science tasks. Each of these WCDs allows for independent groups of 4 Photomultiplier Tubes: one 10-inch at the center and three 8-inch evenly spaced around the center. Every WCD is 7.3 meters in diameter and filled with water up to 4.5 meters and arranged into a hexagonal like pattern [5]. This WCD arrangement allows HAWC to act as a Hodoscope, enabling us to perform a search for nearly horizontal muons (NHM). Extensive Air Shower (EAS) events which cascade radially across a large area of HAWC are a background for our search. We look for NHM in HAWC by identifying events with a linear progression of PMT hits in space and time. The trajectories of these NHM candidates should have a propagation speed near that of light across the array.

The first step in processing the HAWC events is to reject straightforward background events: small EAS events. These events average only 40 or so PMT hits and do not contain PMT hits that are consistent with a particle moving across the array at the speed of light. Nor do the PMT hits satisfy a further requirement using a 2D Hough Transform to find lines indicative of an isolated muon. It is common for a random noise PMT hit within the event to be consistent with some set 
of PMTs within a coincident EAS which then forms an arc or circle of PMTs instead of a linear progression of PMTs. The Hough Transform provides a method to identify lines and shapes such as triangles, rectangles, and circles [6]. Shape identification occurs by assigning a coordinate system to a set of pixels within an image and a voting space. The voting space is a separate parameterized space; for 2-dimensional spaces it is typically a Distance vs. Angular histogram with appropriate binning. The Distance measured is the closest approach for a line, at an angle "A," through a pixel on the image. For a single point, this will look like a sine curve, when all chosen points in the image parameterized, a particular Distance and Angular bin will have votes surpassing a selected cut. This step reduced the data by a factor of approximately 2,000. This initial 2D Hough Transform allows for a reasonably clean sample of events to progress onto a second more rigorous analysis to eliminate the anomalous EAS background events which masquerading as near horizontal muon events not rejected by the first data filter.

The second step of the analysis uses a 3D point cloud iterative Hough Transform, where a good PMT hit in 3-dimensional space $\left(X_{p o s}, Y_{p o s}, \mathrm{c}^{*}\right.$ Time) is equivalent to a two-dimensional pixel. The 3D Hough Transform was developed by Christoph Dalitz et al. at the iPattern institute [7], and uses an icosahedron voting space. The 3D hough transform is utilized twice: first to identify lines and the second as a plane finder as shown in figure 1. We construct the point cloud from the $\mathrm{X}$ and $\mathrm{Y}$ positions of the PMT hits within the event and the $\mathrm{Z}$ component for the hit from the timing of the hit. This iterative algorithm will return multiple lines, if present, with a starting position and direction vector for each line. For each identified line, the original point cloud is rotated such that the direction vector is the new $\hat{Z}$ for that space, and all points are projected onto the $\hat{X}-\hat{Y}$ plane for a second 2D Hough transform. This second rotated analysis will identify any lines that are present, which are potentially an EAS plane. We identify a muon event if a large fraction of the points along the line, $R H_{\text {frac }}$, are associated with the muon candidate at the $\hat{Z}$ axis. An EAS has many points spread along the line indicating the shower plane. Unfortunately, muon bundles are eliminated at this time because they are difficult to distinguish from EAS background events.

\section{Effective Area \& Acceptance}

We calculate the near-horizontal muon effective area and acceptance as a function of arrival direction for HAWC using a simple geometric simulation of HAWC with a "perfect detector" consisting of cylinders located at the positions (including the 0.50 slope of the HAWC platform) of the array of WCDs. In this method, we consider all possible trajectories that intersect rectangular planes perpendicular to arrival directions over the full range of azimuth ( 0 - 360 degrees) and zenith angles from approximately 85 to 92.5 degrees (nearly horizontal). The rectangular plane is $240 \mathrm{~m}$ wide and $60 \mathrm{~m}$ tall divided into a grid with $0.5 \mathrm{~m}$ spacing; this rectangle is sufficiently large to encompass all of HAWC. A trajectory is tested by drawing a ray perpendicular to a grid element to determine the number of hit cylinders with the required intersected track lengths. If a trajectory satisfies the required number of hit cylinders (WCD), the area and acceptance of that grid element is added to the sum to determine the overall effective area and acceptance for that arrival direction. This simple geometry-based effective area calculation provides a means to understand how HAWC's acceptance changes for simple geometric requirements such as intersected tanksegment lengths, the number of hit tanks, and overall track length. To establish an upper bound 
on the effective area and acceptance for each arrival direction, we require each tested trajectory to intersect at least one tank with a minimum intersected length of $10 \mathrm{~cm}$. For the flux measurement that we report, we use the calculated effective area with the requirement of at least 10 tanks each with an intersected length of $3.5 \mathrm{~m}$. Another method using a Geant 4 simulation of the response of HAWC to near-horizontal muons is under development [8].

The observed muon flux as a function of arrival direction does not only depend on the HAWC WCD array. The two neighboring volcanoes [9] provide varying rock overburden depths as a function of arrival direction. We utilize this fact to obtain a measurement of the muon flux as a function of depth. The reconstructed muon trajectories have errors in the arrival direction (azimuth and zenith) determination; this causes uncertainty in the determination of rock overburden depth for an observed muon. This depth uncertainty is especially evident near the edges of the volcanoes or in regions where the depth changes rapidly over a change in angle comparable to the angular uncertainty. In some cases, a particular observed muon arrival direction through a substantial rock overburden depth is more likely to actually have come from a nearby arrival direction with less overburden depth. For the reconstructed arrival direction we calculate the most likely true arrival direction by accounting for the expected reduction in flux as a function of depth and smearing in reconstructed arrival direction angle. This is done by performing a convolution of the flux reduction factor for each nearby arrival direction with a Gaussian smearing of the azimuthal and zenith direction obtained from the Geant 4 simulation. The direction with the highest "probability" from this convolution is then used to determine the depth for the reconstructed arrival direction. This effect is also important in the interiors of the volcanoes away from the edges due to rapid changes in depth with respect to reconstructed arrival direction.

For the majority of Sierra Negra, the depth change is predictable as shown in figure 2 which shows the acceptance correction probability due to depth bias resulting from angular smearing. For our flux measurement, we require that the depth-bias-acceptance correction factor is greater than 0.9 meaning small correction. We can use most of the depths corresponding to arrival directions from Sierra Negra because the correction factor satisfies our requirement. However, Pico de Orizaba retains only the most substantial depth bins at the center of the volcano as also shown in figure 2. The resulting exposure as a function of depth, as seen in figure $3 \mathrm{~b}$, varies significantly among the depth bins due to the variation of rock overburden as a function of arrival directions.

\section{Data}

As mentioned in the previous section on muon identification, we need to separate the NHM signal from the nominal EAS signal for primary HAWC science goals. We have fully analyzed 17 days worth of HAWC triggered data processed through both data filtering processes. An additional 150 days have passed the first analysis step and being processed through the second step. The triggered data rate drops from $25 \mathrm{kHz}$ to a rate of only a few $\mathrm{Hz}$ for potential muon events. The rate of high confidence muon events, a cut on $\mathrm{RH}_{\text {frac }}$ being greater than $99 \%$, reduces the observed muon rate to less than a tenth of a Hz. This cut is required to reduce the contamination from EAS events. The statistics in the higher depth bins becomes very low and dominated by difficult to reduce background events. We have visually inspected these events and identified many EAS-like events when we relax the $R H_{\text {frac }}$ requirement. These background EAS events are proving to be 
challenging to remove as there are few differences between them and real horizontal single muons and muon bundles.

\section{Results}

We presume the near-horizontal muon integral intensity observed at HAWC should be that previously found by underground muon experiments, due to the near-isotropic cosmic ray flux. For the muons which penetrate the rock overburden of a few km.w.e., these are high energy muons produced early in the EAS. The previous experiments which measured this integral intensity of muons for their rock overburden are observing the same population of high energy muons coming from early EAS interactions. We present our preliminary result in figure 4 compared with the previous results. These muons produced early in the EAS event are those which will contribute the most to muon bundles and a future measurement on the cosmic ray composition.

HAWC can accurately reproduce the expected integral intensity of muons for the shallower rock overburden depth bins. For depths more than $5 \mathrm{~km}$.w.e. and up to $10 \mathrm{~km}$.w.e., our fit begins to diverge from the previous results. There are fewer than 20 events for these strict cuts and limited data sample. We visually inspected these events, and they are found to be associated with edge effects in figure 2. If we increase the depth-bias-acceptance correction factor requirement from 0.9, these events are eliminated at the expense of the acceptance in the directions of rapid depth variation. In this case, the 1-2 km.w.e. depth bin has no exposure for the stricter depth-biasacceptance correction factor requirement.

To examine the possible systematic uncertainty in our effective area and acceptance calculation, we compare the geometrical acceptance requiring ten WCDs (blue up triangles), effectively an 80-meter track length, to the perfect detector acceptance calculation. We use the same high confidence $R H_{\text {frac }}$ requirement, and we observe the perfect detector does underestimate the intensity (red squares), but not significantly. The third muon intensity curve (black down triangles) is the result of lowering the $R H_{\text {frac }}$ to a 0.6 confidence fraction. This comparison was made to understand how the intensity changes if we increase the acceptance at the expense of a higher EAS contamination. For depths greater than six km.w.e., nearly all of these events exhibit an EAS plane. For the lower depth bins, a majority of the additional events are muons which previously were cut because of a few random hits with the plane finder which lowers the $\mathrm{RH}_{\text {frac }}$.

Overall, we have demonstrated a good understanding of HAWC operating as a hodoscope for horizontal muons. We are also currently working to improve our EAS background rejection as it eliminates many single muons along with potential muon bundle events. We have inspected some events visually, and found one certain double muon event with a few other possible candidates. The difficulty with the current analysis is that multiple muon event candidates are identified to have a plane with an $R H_{\text {frac }}$ consistent with the EAS backgrounds. The issue here is due to the muons in the bundle being distributed up to 10's of meters in lateral extent. We are looking at the physical parameters of muon bundles which are known from other experiments [4] to implement possible additional cuts, allowing us to relax the $R H_{\text {frac }}$. For example, an estimate of the number of muons in a tightly collimated bundle may be obtained by measuring deposited charge in the WCDs. 


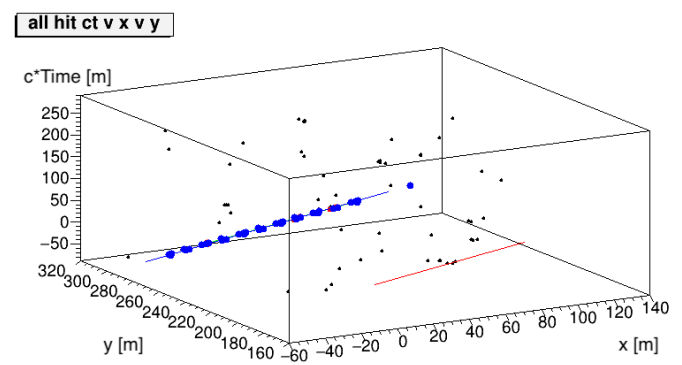

(a) Event point cloud with muon (blue)

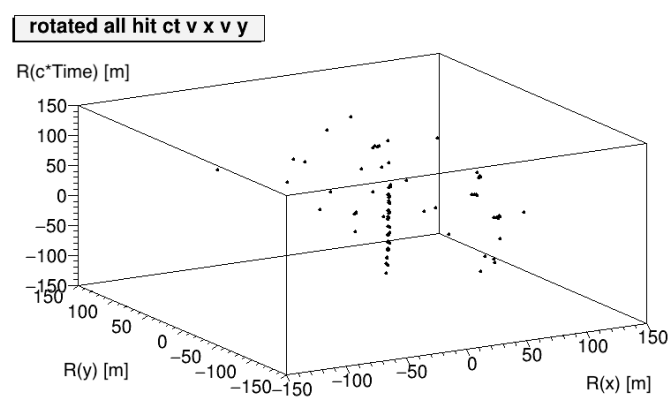

(c) Muon in rotated coordinate point cloud

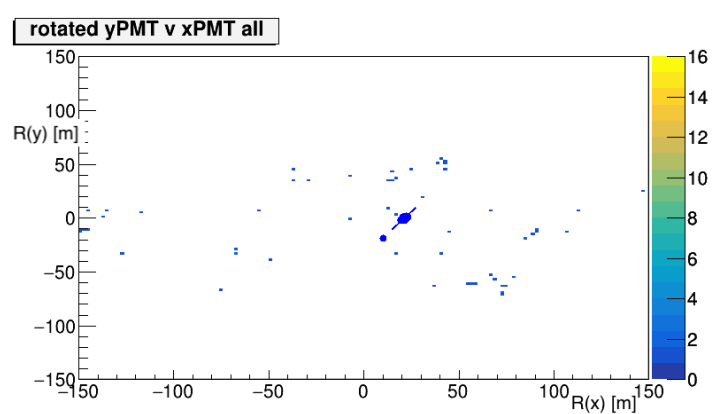

(e) Projected 3D Hough Transform Muon

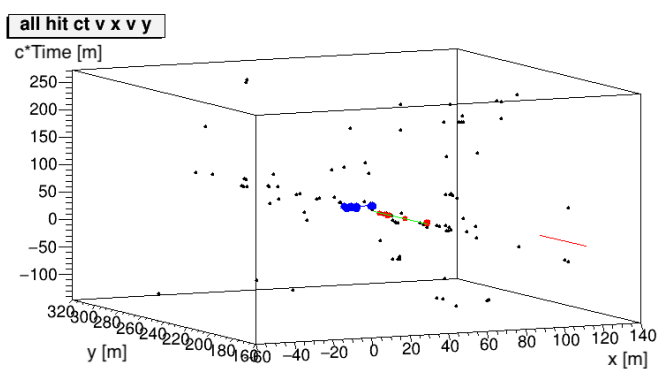

(b) Event point cloud EAS plane

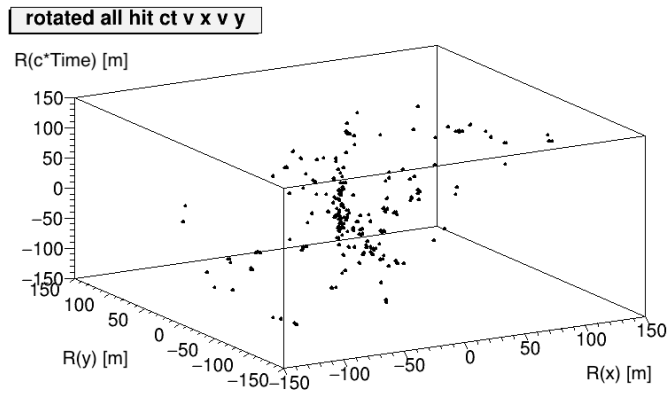

(d) EAS in rotated Coordinate point cloud

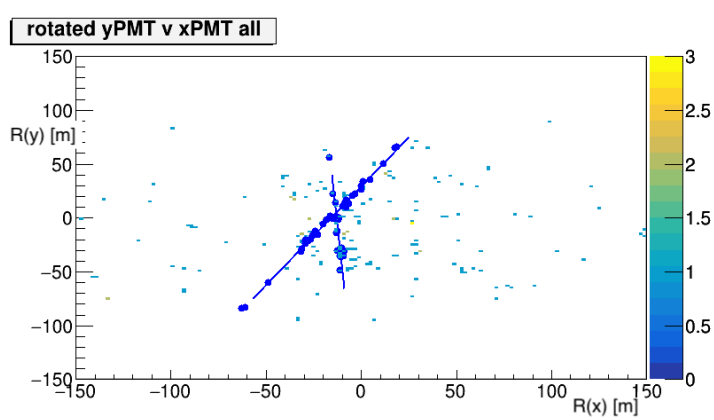

(f) Projected 3D Hough Transform EAS

Figure 1: Left Column: The first 3D hough transform identifies a Line within the original point cloud: the blue points in (a). Subfigure (c) shows how the point cloud is rotated so the Line will align with the $\hat{Z}$ axis. A short line is found in (d) from the projected 3D hough transform and most all points in that line are concentrated at one point. Right Column: an EAS event which is a background event appearing to come from Pico de Orizaba. The Hough Transform points (blue) are stacked and coming out of the page in (b) which is rotated slightly to show the EAS plane in the original point cloud. Figure (d) shows the two rotated point clouds for each of the lines found and (f) shows the two projected 3D hough transform lines indicating an EAS plane. 


\section{Depth Bias Acceptance Correction}

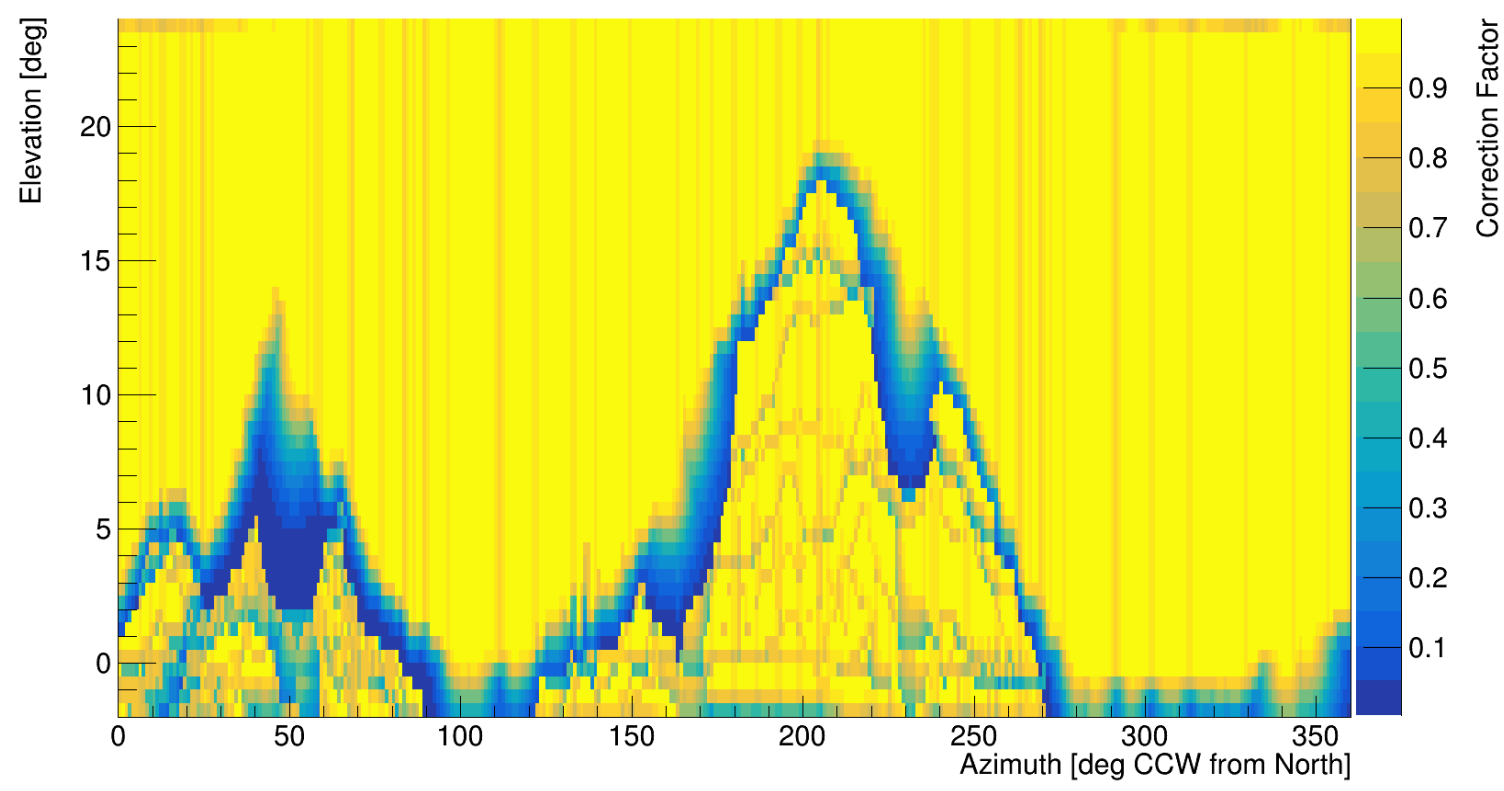

Figure 2: Shown here is the depth-bias-acceptance correction factor. Excluded reconstructed arrival directions are colors less than 0.9 requirement. Pico de Orizaba does have some exposure, however, it is greatly reduced.

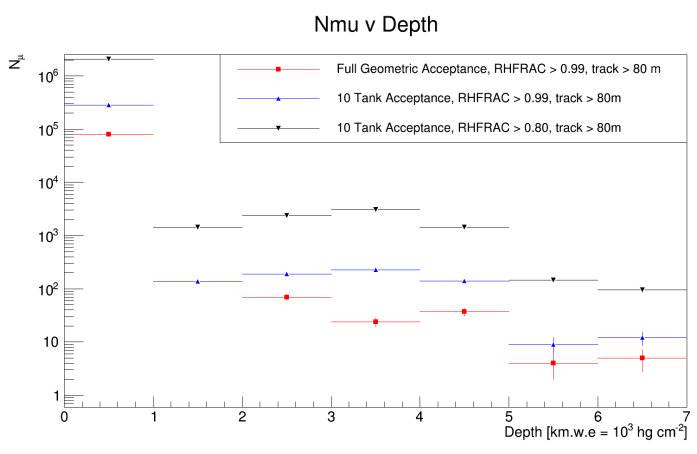

(a) Number of Muons with respect to rock overburden (depth)

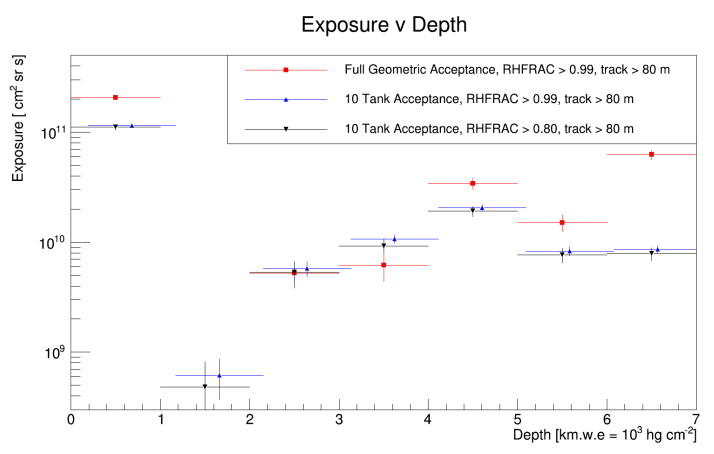

(b) Exposure per depth bin scaled to the current live time.

Figure 3

\section{References}

[1] M. Tanabashi et al. (Particle Data Group), Phys. Rev. D 98, 030001 (2018).

[2] Bugaev, E. V. and Misaki, A. and Naumov, V. A. and Sinegovskaya, T. S. and Sinegovsky, S. I. and Takahashi, N., Atmospheric muon flux at sea level, underground, and underwater, doi 10.1103/PhysRevD.58.054001

[3] H. León Vargas, Prospects of Earth-skimming neutrino detection with HAWC, ICRC 2019, Poster Session 2-111 


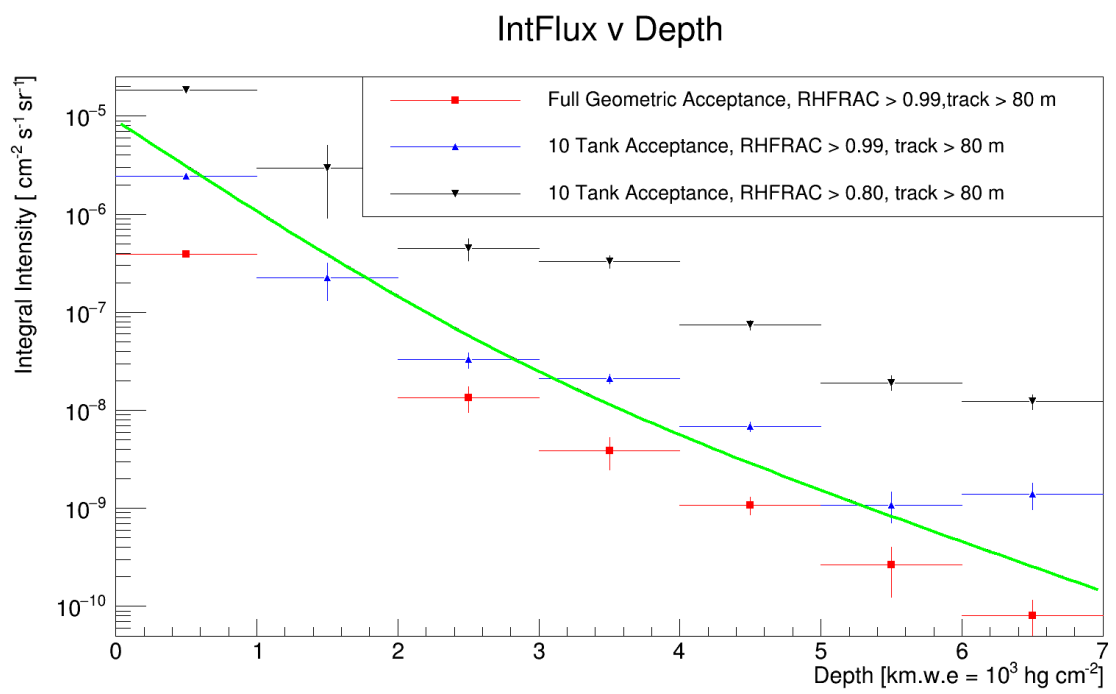

Figure 4: Integral Intensity vs Depth: Red Geometrical Acceptance, Black \& Blue 10-Tank Acceptance with $R H_{\text {frac }}$ cuts of greater than 0.6 and 0.99 respectively. Green line is the Integral Intensity with respect to depth from previous measurements [2].

[4] Oscar Saavedra San Martin, Cosmic ray muon study with the NEVOD-DECOR experiment, $2017 \mathrm{~J}$. Phys.: Conf. Ser. 866012010

[5] A.U. Abeysekara et al., Observation of the Crab Nebula with the HAWC Gamma-Ray Observatory, arXiv:1701.01778 [astro-ph.HE], 10.3847/1538-4357/aa7555

[6] V.F. Leavers, Shape Detection in Computer Vision Using the Hough Transform, 1992, DOI 10.1007/978-1-4471-1940-1

[7] Christoph Dalitz, Tilman Schramke, and Manuel Jeltsch, Iterative Hough Transform for Line Detection in 3D Point Clouds, Image Processing On Line, 7 (2017), pp. 184-196. https://doi.org/10.5201/ipol.2017.208

[8] GEANT4 Collaboration .GEANT4: A simulation toolkit. DOI: 10.1016/S0168-9002(03)01368-8

[9] Instituto Nacional de Estadística y Geografía (INEGI) Continuo de elevaciones mexicano 3.0 (cem 3.0), 2012. URL

http://www.inegi.org.mx/geo/contenidos/datosrelieve/continental/continuoelevaciones.aspx 